\title{
Mechanisch induzierte Apoptose und Nekrose in alveolären Typ-II-Zellen - Beeinflussung durch Captopril und L-Arginin
}

\author{
S. Hammerschmidt \\ H. Kuhn \\ T. Grasenack \\ C. Gessner \\ H. Wirtz
}

\author{
Apoptosis and Necrosis Induced by Cyclic Mechanical Stretching in Alveolar \\ Type-II-Cells - Influence of Captopril and L-Arginine
}

\section{Zusammenfassung}

Hintergrund: Alveoläre Typ-II(ATII)-Zellen sind bei Atmung und Beatmung zyklischen Dehnungen ausgesetzt. Verstärkte Dehnung kann zu akuter Lungenschädigung beitragen. Methode: Diese Arbeit untersucht an ATII-Zellen der Ratte den Effekt dreier Dehnungsmuster (definiert durch Frequenz [min ${ }^{-1}$ ] - Oberflächenzunahme [\%]: S40-13, S60-13, S40-30) auf Parameter von Apoptose, Nekrose und Zellmembranintegrität im Vergleich zu statischen Kontrollen. Das Dehnungsmuster S40 - 13 simuliert physiologische Atmung, die anderen sollen den Einfluss gesteigerter Frequenz und Amplitude demonstrieren. Ergebnisse: Zwischen der Gruppe S40-13 und statischen Kulturen bestanden keine Unterschiede. LDH-Freisetzung und frühapoptotische Zellen waren in den Gruppen S60 - 13 und S40 - 30 im Vergleich zu statischen Kulturen nach 24 h signifikant erhöht (LDH: 0,089 $0,014 \mu \mathrm{g} / \mathrm{ml}$ und $0,177 \pm 0,050 \mu \mathrm{g} / \mathrm{ml}$ gegenüber $0,050 \pm 0,011 \mu \mathrm{g} /$ $\mathrm{ml}$; frühapoptotische Zellen: $17 \pm 3,5 \%$ und $23 \pm 3,1 \%$ gegenüber $9,7 \pm 1,4 \%$ ). Nekrotische Zellen fanden sich nur in der Gruppe S40 - 30 signifikant erhöht ( $13 \pm 2,4 \%$ gegenüber $6,1 \pm 0,9 \%$ in statischer Kultur nach $24 \mathrm{~h})$. Captopril und L-Arginin reduzierten das Ausmaß der Apoptose in der Gruppe S40-30 nahezu auf das Niveau statischer Kulturen, ohne das Ausmaß an Nekrose und

\section{Abstract}

Background: Alveolar type-II(ATII)-cells are exposed to mechanical stretch during breathing and mechanical ventilation. Increased stretch may contribute to lung injury. Methods: The influence of three stretching patterns (characterized by frequency [ $\left.\mathrm{min}^{-1}\right]$ increase in surface area [\%]: S40-13, S60-13, S40-30) on parameters of apoptosis, necrosis and membrane integrity in rat ATII cells was compared with that in static cultures. The S40-13 stretching pattern simulated normal breathing. The other patterns were chosen to study increased amplitude and frequency. Results: There were no significant differences between the S40-13 group and static cultures. LDH release and early apoptotic cells were significantly increased in S60-13 and S40-30 in comparison with static cultures (LDH: $0.089 \pm 0.014 \mu \mathrm{g} / \mathrm{ml}$ and $0.177 \pm 0.050 \mu \mathrm{g} / \mathrm{ml}$ versus $0.050 \pm 0.011 \mu \mathrm{g} / \mathrm{ml}$; early apoptosis: $17 \pm 3.5 \%$ and $23 \pm 3.1 \%$ versus $9.7 \pm 1.4 \%$ ) at $24 \mathrm{~h}$. Necrosis was significantly increased only in the $\$ 40-30$ group ( $13 \pm 2.4 \%$ versus $6.1 \pm 0.9 \%$ in static culture at $24 \mathrm{~h}$ ). Captopril as well as L-Arginine prevented apoptosis and reduced apoptotic cells to static culture levels in the 540 - 30 group but did not influence necrosis and LDH release. Conclusion: Increased mechanical stretch may contribute to lung injury by induction of apoptosis and necrosis in

Institutsangaben

Universität Leipzig, Medizinische Klinik I (Direktor: Prof. Dr. med. J. Schauer)

Anmerkung

Deutsche Fassung von: Hammerschmidt, S, Kuhn H, Grasenack T, Gessner C, Wirtz H.

Apoptosis and Necrosis Induced by Cyclic Mechanical Stretching in Alveolar Type-II-Cells -

Influence of Captopril and L-Arginine.

Am J Respir Cell Mol Biol. 2004 Mar; 30 (3): 396 - 402. Epub 2003 Sep 04.

Zitat nur nach dieser Originalquelle

Widmung

Herrn Prof. Dr. med. Joachim Schauer zum 65. Geburtstag gewidmet.

Danksagung

Diese Arbeit wurde von der Deutschen Forschungsgemeinschaft (Ha 3263/1-1) unterstützt.

Für exzellente technische Assistenz danken wir Frau Konstanze Büttner.

Korrespondenzadresse

Dr. Stefan Hammerschmidt · Medizinische Universitätsklinik I P Pneumologie · Universität Leipzig ·

Johannisallee 32 ·04103 Leipzig · E-mail: stefan.hammerschmidt@t-online.de

Bibliografie

Pneumologie 2004; 58: 222-229 @ Georg Thieme Verlag Stuttgart · New York .

ISSN 0934-8387 · DOI 10.1055/s-2004-818408 
LDH-Freisetzung zu beeinflussen. Schlussfolgerungen: Durch Auslösung von Apoptose und Nekrose an ATII-Zellen kann verstärkte mechanische Dehnung zu akuter Lungenschädigung beitragen. Durch Dehnung mit hoher Amplitude ausgelöste Apoptose kann durch Captopril and L-Arginin verhindert werden.
ATII cells. Apoptosis induced by high amplitude mechanical stretch is prevented by captopril and L-Arginine.

\section{Einleitung}

Alveoläre Typ-II(ATII)-Zellen synthetisieren und sezernieren Surfactant und regenerieren das alveoläre Epithel. Auf diese Weise gewährleisten sie die alveoläre Homöostase. Mechanische Stimuli, wie zyklische Dehnung und Relaxation alveolärer Strukturen während der Atmung, spielen bei der Regulation dieser Prozesse eine entscheidende Rolle [1,2]. In ATII-Zellen stimuliert mechanische Dehnung über kalziumabhängige Signaltransduktion die Phospholipidsekretion [1] und die Expression von Surfactantproteinen [3]. Neben diesen physiologischen Effekten kann mechanische Dehnung von ATII-Zellen auch schädigende Wirkungen entfalten. So führt starke mechanische Dehnung zu Apoptose [4], zu Einrissen der Zellmembran [5] und konsekutivem nekrotischen Zelltod [6,7].

Solche starken mechanischen Dehnungen können beispielsweise im Zusammenhang mit mechanischer Beatmung auftreten. Insbesondere Beatmung mit hohem Atemzugvolumen stellt einen Faktor dar, der akute Lungenschädigung auslösen oder aggravieren kann. So verschlechtert die Beatmung mit hohem im Vergleich zu niedrigem Atemzugvolumen die Prognose beim ARDS (acute respiratory distress syndrome) [8,9]. Verschiedene experimentelle Ergebnisse können diese klinischen Befunde erklären: So konnte gezeigt werden, dass mechanische Dehnung eine Reihe von schädigenden, proinflammatorischen Prozessen, wie die Freisetzung von Prostanoiden und Zytokinen auslöst [2,5,10,11]. Während diese mechanisch induzierte Mediatorfreisetzung als Folge mechanisch induzierter Regulationsprozesse verstanden werden kann, existieren auch experimentelle Daten, die demonstrieren, dass die Einwirkung mechanischer Kräfte direkt zu nekrotischem Zelltod führen kann [6,7]. Die damit verbundene Freisetzung von Bestandteilen des Zytosols zerstörter Zellen kann wiederum eine inflammatorische Reaktion auslösen $[10,12]$.

Apoptose ist der wesentliche Mechanismus, der den Untergang von ATII-Zellen im Zusammenhang mit akuter Lungenschädigung erklärt [13]. Die verminderte Zahl von ATII-Zellen führt zu verminderter Surfactantproduktion und -sekretion sowie zu einem gestörten Surfactantrecycling und ist eine Erklärung für den bei akuter Lungenschädigung vorliegenden Surfactantmangel [14]. Daneben ist infolge verstärkter Apoptose von ATII-Zellen auch die Regeneration des alveolären Epithels gestört.

In den bisherigen Arbeiten zu mechanisch induzierter Apoptose und Nekrose an ATII-Zellen wurden einerseits nur unphysiologische Dehnungsmuster (3 Dehnungen/min) verwendet [4], andererseits untersuchten diese Arbeiten nur einzelne Dehnungsmuster $[15,16]$ oder untersuchen ATII-Zellen nach fünf Tagen in
Kultur unter der Annahme, dass diese Zellen dann eher alveoläre Typ-I-Zellen repräsentieren [6,7].

Ziel dieser Arbeit ist es, den Einfluss von Dehnungsfrequenz, Dehnungsamplitude und Dauer der zyklischen Dehnung auf die Auslösung mechanisch induzierter Apoptose und Nekrose zu charakterisieren. Weiterhin sollten Substanzen mit zu erwartenden antiapoptotischem Effekt hinsichtlich ihrer Wirkung auf die mechanisch induzierte Apoptose und Nekrose untersucht werden.

\section{Methodik}

\section{Präparation von ATII-Zellen}

ATII-Zellen wurden mit der von Dobbs u. Mitarb. 1986 [17] entwickelten und in verschiedenen Vorarbeiten beschriebenen Methode $[1,18,19]$ präpariert. Dabei werden ATII-Zellen aus den Lungen männlicher Sprague-Dawley-Ratten (150-200 g) mittels Elastase-Verdau und selektiver Adhärenz auf IgG-beschichteten Platten präpariert. Zum Zeitpunkt des Ausplattierens waren $89 \pm 3,4 \%$ der Zellen vitale ATII-Zellen (nachgewiesen durch modifizierte Papanicolaou-Färbung).

Die ATII-Zellen werden auf die zentralen Bereiche (ca. $1,5 \mathrm{~cm}$ Durchmesser) der mit Fibronectin beschichteten, dehnbaren Silikonmembranen (Bioflex ${ }^{\circledR}$, Flexcell International, Hillsborough, NC, USA) ausplattiert. Dabei werden auf 6-well-Platten $10^{6} \mathrm{Zel}-$ len je Well in Dulbeccos modified Eagle's medium (DMEM) mit $10 \%$ fetalem Kälberserum (FCS), $1 \%$ w/v Gentamicin und 1\% Glutamine ausplattiert. Nach einer Adhärenzperiode von $22 \mathrm{~h}$ wird das Medium durch DMEM mit 2\% FCS ersetzt. Mit diesen Platten wurden die Experimente durchgeführt.

\section{Versuchsprotokoll}

Die ATII-Zellen auf den Bioflex ${ }^{\circledR}$ Platten wurden mit einem FX $4000 \mathrm{~T}^{\mathrm{TM}}$ Flexercell ${ }^{\circledR}$ Tension Plus ${ }^{\mathrm{TM}}$ System (Flexcell International, Hillsborough, NC, USA) zyklischer Dehnung ausgesetzt. Eines von drei Dehnungsmustern wurde zufällig gewählt und über die gesamte Versuchszeit von $24 \mathrm{~h}$ angewendet. Zellen und Überstände wurden zu den Zeiten $\mathrm{t}=0 \mathrm{~min}$ (vor Beginn der Dehnung - Ausgangswerte), $\mathrm{t}=12 \mathrm{~h}, \mathrm{t}=18 \mathrm{~h}$ und $\mathrm{t}=24 \mathrm{~h}$ gesammelt.

Die Dehnungsmuster wurden durch Frequenz und Amplitude (Oberflächenzunahme der kalibrierten Silikonmembran) definiert. Die Membrandehnung wurde mit negativem Druck kalibriert und während der gesamten Versuchsdauer überwacht.

Die von uns genannten Zahlen der Oberflächezunahme entsprechen nicht denen, die in der Software des Dehnungsgerätes gewählt werden können, sondern der realen, tatsächlich im Versuch durchgängig anhand des negativen Drucks während jeder 
Dehnung berechneten Oberflächenänderungen der Membran. In dieser Berechnung wurden die Eigenschaften der verwendeten Membranen ebenso wie die der jeweils verwendeten Grundplatte des Dehnungsgerätes berücksichtigt.

ATII-Zellen auf identisch beschichteten Silikonmembranen wurden nicht zyklisch gedehnt und dienten als Kontrollen (statische Kultur).

\section{Dehnungsmuster}

Die Dehnungsmuster wurden so definiert, dass in dem Modell der ATII-Zellen der Ratte der Einfluss von Dehnungsfrequenz und -amplitude charakterisiert werden kann. In der Rattenlunge kommt es zu einer Zunahme der Basalmembranoberfläche ab einer Inflation von mehr als ca. 40\% TLC [20]. Veränderung des Volumens unterhalb dieser Größenordnung (d.h. unterhalb der funktionellen Residualkapazität [FRC]) führen wahrscheinlich nur zu einer Entfaltung der Basalmembran und/oder zu einer Eröffnung kollabierter Alveolen und nicht zur Dehnung der Basalmembran. Um die Dehnungsamplitude festzulegen, orientierten wir uns an dem Zusammenhang zwischen Veränderung der Basalmembranoberfläche und dem Lungenvolumen, wie er durch Tschumperlin u. Mitarb. 1999 beschrieben wurde [20]. Dabei entsprechen Oberflächenänderungen der Basalmembran von $13 \%$ und $30 \%$ im Bezug zur Oberfläche bei $42 \%$ TLC etwa Lungenblähungen bis 75 und 100\% TLC (Funktion in Abb. 3A [20]). Die so gewählten Dehnungsamplituden entsprechen also zum einen etwas mehr als der Atmung mit normalem Atemzugvolumen und zum anderen ziemlich genau der maximalen Inspiration. Um den Einfluss der Frequenz zu charakterisieren wurden 40 und 60 min $^{-1}$ gewählt. Aus technischen Gründen war es nicht möglich, eine Dehnungsfrequenz von $60 \mathrm{~min}^{-1}$ mit einer Amplitude von $30 \%$ zu verbinden. Daher nutzten wir drei Dehnungsmuster [S], die durch Frequenz [ $\left.\mathrm{min}^{-1}\right]$ und Amplitude [\% Oberflächenzunahme] definiert sind: $S 40-13, S 60-13$ und $S 40-30$.

Um auszuschließen, dass die dehnbaren Membranen durch Dehnung eine zytotoxische Verbindung freisetzen, welche selbst Apoptose oder Nekrose auslösen könnte, wurde folgendermaßen verfahren: Kulturmedium wurde auf Platten gegeben und ohne Zellen zyklischer Dehnung über $24 \mathrm{~h}$ ausgesetzt (S40-30). Dieses Medium wurde dann auf Zellen in statischer Kultur gegeben und mit dem Medium von statischen Membranen verglichen. Es ergab sich kein Unterschied hinsichtlich der Induktion von Apoptose und Nekrose.

\section{LDH-Messung}

Die Konzentration der freigesetzten Laktatdehydrogenase (LDH) wurde in den Überständen gemessen (Cytotoxicity Detection Kit [Roche Diagnostics GmbH, Mannheim, Germany]). Die Eichkurve (Standard-LDH von Sigma-Aldrich GmbH, Deisenhofen, Germany) wurde zwischen 2 und $0,016 \mu \mathrm{g} / \mathrm{ml}$ gewählt.

\section{Apoptose und Nekrose}

Annexin-V-Bindung und PI-Färbung: Die Zellen wurden mit Trypsin von den dehnbaren Membranen gelöst. In die Analyse wurden adhärente und nichtadhärente Zellen einbezogen. Die Zellen wurden mit FITC-konjugiertem Annexin V und Propidiumiodid (PI) gefärbt (Apoptosis Detection Kit [R\&D Systems, Minneapolis, MN, USA]), und durchflusszytometrisch analysiert (Epics XL,
Beckman-Coulter). Jeder Datenpunkt repräsentiert $n=4$ Versuche mit Dreifachmessung.

Anreicherung von Oligonucleosomen: In Überständen wurde die Anreicherung von Oligonucleosomen gemessen (Cell Death Detection ELISA plus ${ }^{\circledR}$ [Roche, Mannheim, Germany]). Der Anleitung des Herstellers folgend wurde ein Anreicherungsfaktor bestimmt, der die Relation zwischen gedehnten Zellen und statischen Kontrollen angibt. Jeder Datenpunkt repräsentiert $n=3$ Versuche mit Dreifachmessung.

Caspase-8-Aktivität: Im Zellhomogenat wurde die Aktivität der Caspase-8 kolorimetrisch bestimmt (R\&D Systems, Minneapolis, MN, USA). Rekombinante Caspase-8 (R\&D systems) wurde als Standard verwendet. Jeder Datenpunkt repräsentiert $n=3$ Versuche mit Dreifachmessung.

\section{Einfluss von Captopril und L-Arginin}

In einer eigenständigen Gruppe von Experimenten wurden die Effekte des ACE-Hemmers Captopril (CAP, Endkonzentration $50 \mathrm{ng} / \mathrm{ml}$ ) und des Substrates der NO-Synthase L-Arginin (LAR, Endkonzentration $1 \mathrm{mM}$ ) auf die mechanisch induzierte Apoptose und Nekrose charakterisiert. In diesen Experimenten wurde das Dehnungsmuster verwendet, welches die höchste Rate an apoptotischen Zellen erzielte. Als Kontrolle dienten statische Kulturen mit Zugabe der zu testenden Substanzen und gedehnte Kulturen ohne diese Substanzen. Jeder Datenpunkt repräsentiert $n=6$ Versuche mit Dreifachmessung.

\section{Angiotensin II ELISA}

Die Überstände gedehnter Zellen (alle drei Dehnungsmuster) wurden nach $24 \mathrm{~h}$ auf Angiotensin II analysiert (SpiBio, Massy, Cedex, France; $n=3$ Zellpräparationen, Dreifachmessungen).

\section{Statistik}

Für die Vergleiche zwischen den Versuchsgruppen wurde der $\mathrm{t}$-Test mit Bonferoni-Korrektur für Mehrfachtestung verwendet. Die Ergebnisse sind als Mittelwert \pm Standardabweichung angegeben.

\section{Ergebnisse}

\section{Laktatdehydrogenase}

Die LDH-Konzentration wurde in den Überständen aller Versuchsgruppen gemessen (Abb.1). In den Überständen der statischen Kontrollen stieg die LDH-Konzentration über die Zeit leicht, auf ein Maximum von $0,050 \pm 0,011 \mu \mathrm{g} / \mathrm{ml}$ nach $24 \mathrm{~h}$, an Die LDH-Freisetzung in der S40-13 Gruppe $(0,059 \pm 0,011 \mu \mathrm{g} / \mathrm{ml}$ nach $24 \mathrm{~h}$ ) unterschied sich nicht signifikant von der in statischen Kulturen. Die LDH-Freisetzung in den Versuchsgruppen S60- 13 und S40-30 war dagegen nach 12,18 und $24 \mathrm{~h}$ signifikant höher als in den statischen Kulturen (S60-13:0,089 \pm $0,014 \mu \mathrm{g} / \mathrm{ml} \quad \mathrm{p}<0,0001$ und $\quad \mathrm{S} 40-30: \quad 0,177 \pm 0,050 \mu \mathrm{g} / \mathrm{ml}$, $\mathrm{p}<0,0001$ nach $24 \mathrm{~h}$ ). Am größten war die LDH-Freisetzung in der Versuchsgruppe mit der großen Dehnungsamplitude $(S 40-30, p=0,0002$ gegenüber $S 40-13$ und $p=0,031$ gegenüber S60-13 nach $24 \mathrm{~h}$ ). 

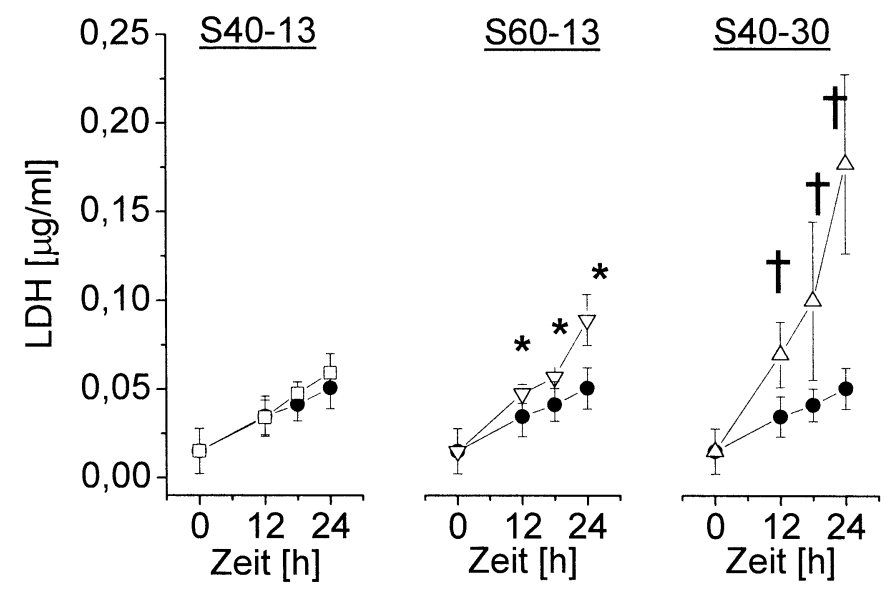

Abb. 1 LDH-Freisetzung. LDH-Konzentration in den Überständen zyklisch gedehnter Zellen (offene Symbole) und statischer Kulturen (schwarze Punkte). Die Diagramme zeigen den Vergleich der Versuchsgruppen $\mathrm{S} 40-13, \mathrm{~S} 60-13$ und $\mathrm{S} 40-30$ mit den statischen Kulturen. *: $p<0,05$ im Vergleich zu statischen Kulturen; $\dagger: p<0,05$ im Vergleich zu statischen Kulturen und zu den Gruppen S40-13 und S60-13.

\section{Annexin-V-Bindung und PI-Färbung}

Die Ergebnisse der Durchflusszytometrie sind in Abb. 2 zusammengefasst. Annexin-V-negative/PI-negative Zellen (Abb. 2A) wurden als vitale Zellen betrachtet. Der Prozentsatz dieser Zellen unterschied sich in den Versuchsgruppen S40-13 und S60-13 nicht signifikant von dem in statischer Kultur. Ein signifikanter Rückgang der vitalen Zellen über die Versuchszeit konnte dagegen unter dem Dehnungsmuster S40-30 beobachtet werden ( $55 \pm 12$ gegenüber $81 \pm 2,4 \% \mathrm{p}=0,0002$ nach $24 \mathrm{~h}$ ). Diese Abnahme der Zahl vitaler Zellen konnte mit einer Zunahme AnnexinV-negativer/PI-positiver (nekrotischer) Zellen und mit einer Zunahme Annexin-V-positiver/PI-negativer (frühapoptotischer) sowie Annexin-V-positiver/PI-positiver (spätapoptotischer/nekrotischer) Zellen erklärt werden (Abb. 2B-D).

Im Vergleich zur statischen Kultur signifikant erhöht fand sich der Prozentsatz der frühapoptotischen Zellen (Abb. 2B) sowohl in der Versuchsgruppe $S 60-13$ ( $17 \pm 3,5 \%$ gegenüber $9,7 \pm 1,4 \%$, $\mathrm{p}=0,037$ nach $24 \mathrm{~h}$ ) als auch in der Versuchsgruppe $\mathrm{S} 40-30$ ( $21 \pm 3,2 \%$ gegenüber $13 \pm 1,7 \%, \mathrm{p}=0,035$ nach $18 \mathrm{~h}$ und $23 \pm$ $3,1 \%$ gegenüber $9,7 \pm 1,4 \%, p=0,0044$ nach 24 h). Der Prozentsatz der nekrotischen Zellen (Abb. 2D) unterschied sich zwischen statischen Kulturen und zyklisch gedehnten Zellen der Versuchsgruppen S40-13 und S60-13 nicht signifikant. Unter dem Dehnungsmuster mit hoher Amplitude (S40-30) dagegen war ein zweigipfeliger Anstieg der nekrotischen Zellen mit einem frühen Gipfel nach $12 \mathrm{~h}$ und einem zweiten Gipfel nach $24 \mathrm{~h}(18 \pm 2,2 \%$ gegenüber $6,6 \pm 1,1 \%, p=0,0002$ und $13 \pm 2,4 \%$ gegenüber $6,1 \pm$ $0,9 \%, p=0,0034)$ zu verzeichnen. Im Gegensatz dazu stieg der Prozentsatz frühapoptotischer Zellen beginnend nach $18 \mathrm{~h}$ kontinuierlich an. Die Zahl der spätapoptotischen/nekrotischen Zellen (Abb. 2C) stieg nach $12 \mathrm{~h}$ an und erreichte ein Maximum nach $18 \mathrm{~h}$.

Diese Ergebnisse legen nahe, dass in dieser Versuchsgruppe der Zelluntergang in zwei Phasen abläuft. Einer frühen Phase nekrotischen Zelltods folgt eine Phase, die vor allem durch apoptotischen Zelltod charakterisiert ist.

\section{Anreicherung von Oligonucleosomen}

Der Anreicherungsfaktor für Oligonucleosomen in den Überständen gedehnter Zellen im Vergleich zu statischen Kulturen ist in Abb. 3 wiedergegeben. Die Versuchsgruppen mit niedriger Dehnungsamplitude (S40-13 und S60-13) unterschieden sich in diesem Anreicherungsfaktor nicht voneinander. Im Vergleich zu diesen beiden Versuchsgruppen kam es jedoch in den Versuchen mit hoher Dehnungsamplitude ( $540-13)$ zu einer signifikant stärkeren Anreicherung von Oligonucleosomen im Überstand.

\section{Caspase-8-Aktivität}

Die in den Zelllysaten gemessene Aktivität der Caspase-8 aller Versuchs- und Kontrollgruppen ist in der Abb. 4 zusammengefasst. Nur in der Versuchsgruppe mit hoher Dehnungsamplitude wurde ein signifikanter Unterschied zwischen den Experimenten mit gedehnten Zellen und statischen Kulturen gefunden.

\section{Angiotensin II}

Mit dem verwendeten Assay (Angiotensin ELISA, SpiBio, Massy, Cedex, France) konnte Angiotensin II nicht nachgewiesen werden.

\section{Einfluss von Captopril und L-Arginin}

Die Abb. 5 zeigt, wie Captopril und L-Arginin die mechanisch induzierte LDH-Freisetzung, Apoptose und Nekrose in der Versuchsgruppe $540-30$ beeinflussen. Beide Substanzen veränderten die mechanisch induzierte LDH-Freisetzung und Nekrose nicht, verhinderten aber die mechanisch induzierte Apoptose nahezu vollständig, so dass der Prozentsatz der frühapoptotischen Zellen in den Versuchen mit diesen Verbindungen nahezu die Werte der Kontrollgruppen erreichte $(10,7 \pm 2,2 \%$ und 10,5 \pm $2,5 \%$ in den Gruppen CAP und LAR). Ebenso verringerten Captopril und L-Arginin die Zahl der spätapoptotischen/nekrotischen Zellen fast auf das Niveau der statischen Kulturen. Die Zahl der vitalen Zellen wurde durch Captopril und L-Arginin dementsprechend deutlich gesteigert auf $77 \pm 2,6 \%$ und $76 \pm 3,8 \%$ nach $24 \mathrm{~h}$ (statische Kulturen: $82 \pm 3 \%$ ).

\section{Diskussion}

Diese Studie untersucht an ATII-Zellen die Effekte zyklischer mechanischer Dehnung auf LDH-Freisetzung, apoptotischen und nekrotischen Zelltod. Wir wählten zunächst ein Dehnungsmuster, dass die mechanische Stimulation dieser Zellen unter physiologischer Atmung simulieren sollte ( $\mathrm{S} 40$ - 13). Eine Frequenz von 40/min entspricht der Atemfrequenz der Ratte in Ruhe, und eine Oberflächenzunahme der Zellmembran von $13 \%$ entspricht ungefähr einer Inspiration von der FRC bis zu 75\% TLC [20]. Die unter diesem Dehnungsmuster freigesetzte LDH überschritt nicht die in statischen Kontrollen freigesetzte und gewöhnlich in Zellkultur gefundene LDH-Freisetzung. Ebenso fanden sich unter diesem Dehnungsmuster die Zahl vitaler, apoptotischer und nekrotischer Zellen nicht verschieden von den Werten statischer Kulturen.

Ein weiteres Dehnungsmuster wurde so gewählt, dass die Dehnungsamplitude $(30 \%)$ der Oberflächen der Zellmembran bei maximaler Inspiration (100\% TLC) entspricht. Dieses Dehnungsmuster führte - beginnend zu $12 \mathrm{~h}$ - zu einer signifikanten $\mathrm{Zu}$ - 


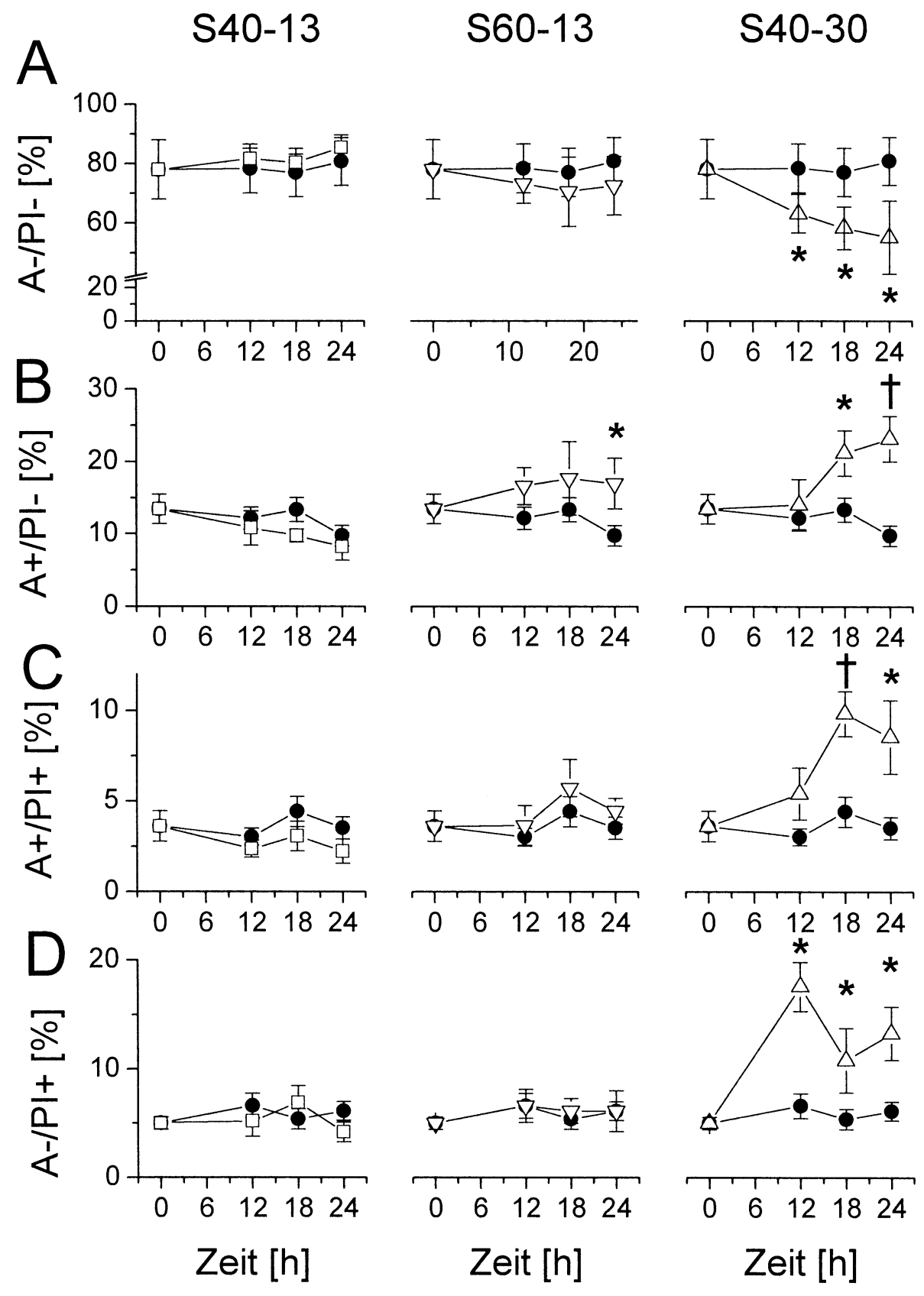

Abb. 2 Annexin-V-Bindung und PI-Färbung. Durchflusszytometrische Daten von zyklisch gedehnten Zellen (offene Symbole) und statischen Kulturen (schwarze Punkte). Die Diagramme zeigen den Vergleich zwischen den Versuchsgruppen $\$ 40-13$, S60 - 13, S40 - 30 und statischen Kulturen. Reihe A zeigt den Prozentsatz vitaler Zellen, Reihe B den frühapoptotischer Zellen, Reihe $C$ den spätapoptotische/nekrotischer Zellen und Reihe $\mathrm{D}$ den nekrotischer Zellen.

$*$ : $p<0,05$ im Vergleich zu statischen Kulturen und zur Gruppe $\$ 40-13 ; \dagger: p<0,05 \mathrm{im}$ Vergleich zu statischen Kulturen und zu den Gruppen S40-13 und S60-13. nahme der LDH-Freisetzung und zu einem signifikanten Anstieg apoptotischer und nekrotischer Zellen. Entsprechend sank die Zahl der vitalen Zellen über die Zeit. Der zeitliche Verlauf der LDH-Freisetzung, der Marker für Apoptose und Nekrose lässt vermuten, dass zwei Mechanismen zu dem beobachteten Rückgang der vitalen Zellen führen: Bereits nach $12 \mathrm{~h}$ kam es zu einem frühen Anstieg nekrotischer Zellen, während die Zahl der apoptotischen Zellen zu diesem Zeitpunkt nur moderat angestiegen war. Nach 18 und $24 \mathrm{~h}$ dagegen steigt die Anzahl der apoptotischen Zellen deutlich an, während die Zahl der nekrotischen Zellen nach $18 \mathrm{~h}$ zurückgeht - die erste Nekrose-Phase ist offensichtlich abgelaufen. Nach $24 \mathrm{~h}$ führt offensichtlich die vermehrt ablaufende Apoptose zu einem zweiten Anstieg toter Zellen. Das Modell des mechanisch induzierten "membrane stress failure“ lässt sich mit unserer Beobachtung gut in Einklang bringen: Zellen, die nach Einrissen der Zellmembran durch mechanische Dehnung nicht suffizient verschließen und den mit dem Einriss verbundenen Kalziumeinstrom kompensieren können, werden nekrotisch. In anderen Zellen, in denen nur kurzzeitig mechanisch induzierte Einrisse der Zellmembran bestanden haben, können die dadurch ausgelösten Signalprozesse zur Induktion von Apoptose führen.

Eine gesteigerte Dehnungsfrequenz (von 40 auf 60/min) war verglichen mit einer gesteigerten Amplitude - klar der weniger traumatische mechanische Stimulus. In dieser Versuchsgruppe (S60 - 13) fanden wir die Zahl der vitalen Zellen nach $24 \mathrm{~h}$ nur gering, nicht signifikant reduziert. Lediglich die Zahl der frühapoptotischen Zellen (gemessen als Annexin-V-Bindung) war nach $24 \mathrm{~h}$ signifikant angestiegen, und die LDH-Freisetzung war im Vergleich zum S40-13 Dehnungsmuster während aller Messpunkte erhöht. Mit dem zweiten, zum Nachweis früher Apoptose eingesetzten Assay, der Bestimmung der Caspase-8-Aktivität, konnte dieser Anstieg der Annexin-V-positiven/PI-negativen Zel- 


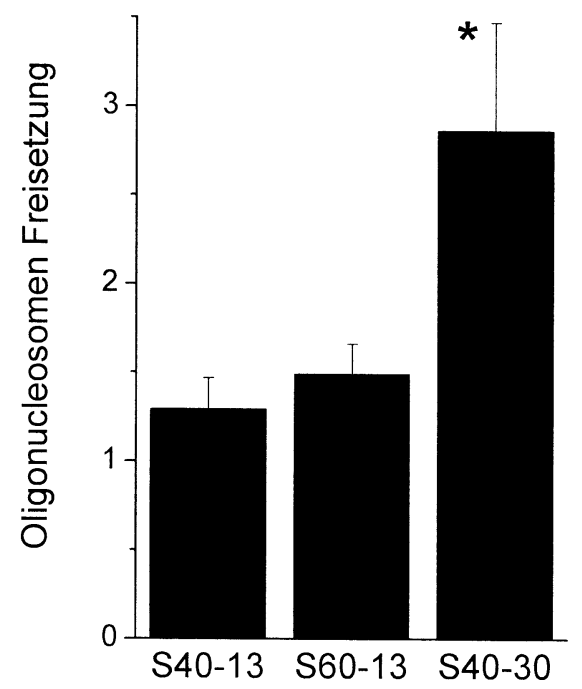

Abb. 3 Freisetzung von Oligonucleosomen. Die Freisetzung von Oligonucleosomen in die Überstände von Zellen während zyklischer Dehnung über $24 \mathrm{~h}$ wurde mit einem ELISA, welcher histonassoziierte DNA erkennt, gemessen. Die Konzentration der histonassoziierten DNA in Überständen gedehnter Zellen wurde zu den Konzentrationen statischer Kontrollen ins Verhältnis gesetzt. Dieser Anreicherungsfaktor ist dargestellt (relative Einheiten). ${ }^{*} \mathrm{p}<0,05$ im Vergleich zu den Gruppen S60- 13 und $\$ 40-13$.

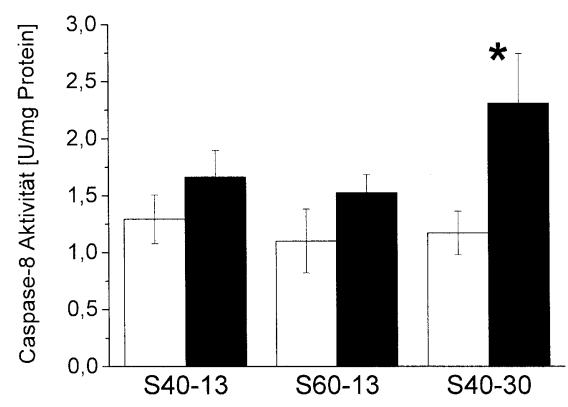

Abb. 4 Caspase-8-Aktivität. Die Aktivität der Caspase-8 wurde in den Lysaten der Zellen nach 24 h zyklischer (schwarze Säulen) Dehnung oder statischer Kultur (offene Säulen) gemessen und als Mittelwert \pm Standardfehler angegeben. ${ }^{*}: \mathrm{p}<0,05$ im Vergleich zur korrespondierenden statischen Kontrollgruppe.

len nach $24 \mathrm{~h}$ Stunden in dieser Versuchsgruppe nicht reproduziert werden. Eine Erklärung für die abweichenden Ergebnisse der beiden Tests kann eine geringere Empfindlichkeit der Caspase-8-Bestimmung sein, da die Aktivität dieses Enzyms unabhängig von Apoptose reguliert wird [21]. Erwähnenswert ist, dass es unter dem Dehnungsmuster mit erhöhter Frequenz nicht zu einem Anstieg der nekrotischen Zellen gekommen ist. Auch dieser Befund spricht dafür, dass dehnungsinduzierte Membraneinrisse den wesentlichen schädigenden Mechanismus darstellen.

Wie bereits dargestellt, entspricht eine Zunahme der Zellmembranoberfläche um $30 \%$ ungefähr einer Inspiration von der FRC bis zu $100 \%$ TLC [20]. Dieses Ausmaß an alveolärer Dehnung kann erreicht und sogar überschritten werden unter mechanischer Beatmung mit hohen Atemzugvolumina und bei schwerer akuter Lungenschädigung selbst unter Beatmung mit niedrigen Atemzugvolumina, wenn Derekruitment großer Lungenanteile die belüfteten Areale der Lunge deutlich reduziert hat. Die Frei-
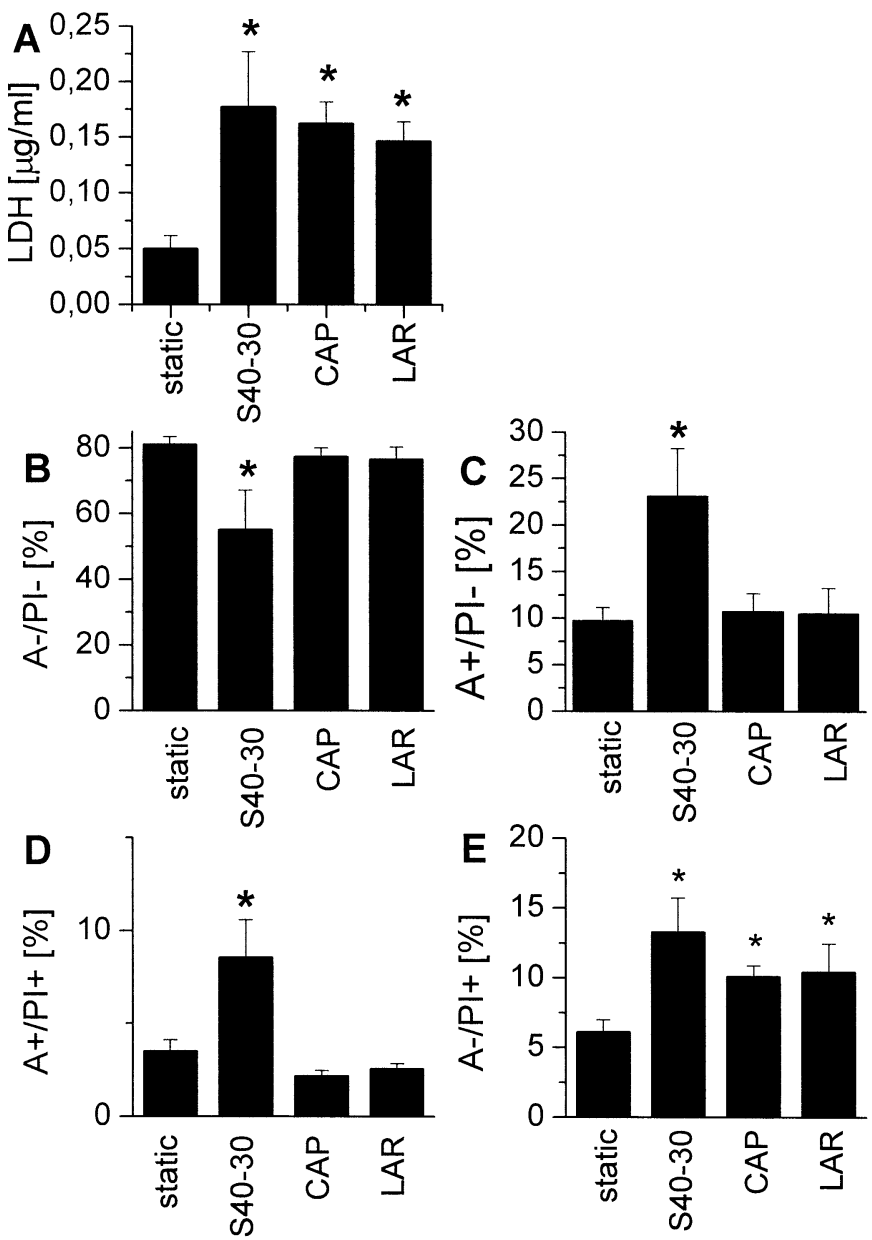

Abb. 5 Effekte von Captopril and L-Arginin. Die Effekte von Captopril (CAP) und L-Arginin (LAR) auf LDH-Freisetzung (A), vitale (B), frühapoptotische (C), spätapoptotische/nekrotische (D) und nekrotische Zellen (E) sind zusammengefasst. Statische Kontrollen werden mit zyklisch gedehnten ATII-Zellen ( 540 - 30, 24 h) ohne oder mit Zusatz von $50 \mathrm{ng} /$ $\mathrm{ml}$ Captopril (CAP) oder $1 \mathrm{mM}$ L-Arginin (LAR) verglichen. ${ }^{*} \mathrm{p}<0,05 \mathrm{im}$ Vergleich zur statischen Kontrolle.

setzung von Bestandteilen des Zytosols aus nekrotischen Zellen kann proinflammatorisch wirken und dadurch den Prozess der akuten Lungenschädigung unterhalten und verstärken $[10,12]$. Jedoch nicht nur Nekrose, sondern auch Apoptose von ATII-Zellen trägt zu akuter Lungenschädigung bei. Durch intratracheal applizierte, Fas-aktivierende monoklonale Antikörper hervorgerufene Apoptose führt bei Mäusen zu akuter Lungenschädigung [22]. Nekrose und Apoptose von ATII-Zellen scheint eine wesentliche Verbindung zwischen mechanischem Stress und der Verstärkung akuter Lungenschädigung darzustellen.

In dieser Arbeit haben wir verschiedene Methoden eingesetzt, um die Vitalitätsparameter von ATII-Zellen zu charakterisieren: Die durchflusszytometrische Messung von Annexin-V-Bindung und PI-Färbung ist eine weit verbreitet angewendete Methode, um die Anzahl vitaler, frühapoptotischer, spätapoptotischer/nekrotischer und nekrotischer Zellen zu quantifizieren. Darüber hinaus wählten wir zwei zusätzliche Verfahren, um unsere durchflusszytometrischen Daten zu unterstreichen: Die Bestimmung der Caspase-8-Aktivität wurde gewählt, weil dieses Enzym sehr proximal in der Caspasen-Kaskade lokalisiert ist und daher ein 
frühes Stadium der Apoptose anzeigt. Limitiert wird diese Methode, weil dieses Enzym durch zahlreiche endogene regulatorische Proteine unabhängig von Apoptose beeinflusst wird [21]. Die Anreicherung von Oligonucleosomen im Überstand kann das Ergebnis von prälytischer DNA-Fragmentierung mit nachfolgender Freisetzung durch zelluläre Lysis in der späten Phase der Apoptose sein. Allerdings kann sie auch das Ergebnis von postlytischer DNA-Fragmentierung sein. Deshalb entspricht die Information dieser Messungen jener, die die Fraktion der durchflusszytometrisch gemessenen Annexin-V-positiven/PI-positiven Zellen liefert.

Der mechanisch induzierte Anstieg an spätapoptotischen/nekrotischen und an frühapoptotischen Zellen unter dem Dehnungsmuster S40-30 ist durch beide getesteten Pharmaka, durch den ACE-Hemmer Captopril und das NOS-Substrat L-Arginin, nahezu vollständig inhibierbar. Die Zahl der nekrotischen Zellen dagegen bleibt von der pharmakologischen Intervention unbeeinflusst. Captopril und L-Arginin hemmen offensichtlich die mechanisch induzierte Apoptose.

Der Prozentsatz apoptotischer Zellen war in unserer Arbeit höher als in einer vorausgegangenen Arbeit, die ein ähnliches experimentelles Modell nutzt [16]. Neben zahlreichen experimentellen Faktoren kann vor allem die Zusammensetzung des Mediums solche Unterschiede erklären. Die Autoren dieser Studie berichten ca. 5\% apoptotische Zellen unter statischen Bedingungen. Apoptose wurde in dieser Studie jedoch durch DNA-Kondensation mit dem Farbstoff Hoechst 33258, der eher späte Stadien der Apoptose detektiert, nachgewiesen. Im Gegensatz dazu verwendeten wir mit Annexin-V-Bindung einen Marker eines frühen Apoptosestadiums. Außerdem war in unseren Experimenten die FCS-Konzentration wesentlich niedriger (2 gegenüber 10\%). Dies deckt sich gut mit unserer Erfahrung, dass die FCS-Konzentration des Mediums sowohl in statischen Kontrollen als auch in zyklisch gedehnten Kulturen das Ausmaß der Apoptose erheblich bestimmt (Hammerschmidt, u. Mitarb. unpublizierte Daten). Der von Edwards u. Mitarb. [16] berichtete maximal dreifache Anstieg des Prozentsatzes apoptotischer Zellen unter zyklischer Dehnung deckt sich sehr genau mit unseren Ergebnissen. Der relative Effekt mechanischer Dehnung ist daher zwischen beiden Studien gut vergleichbar.

Zwischen 40 und 50\% nichtvitale Zellen nach nur einer Stunde zyklischer Dehnung (15/min, 25\% Zunahme der Zellmembranoberfläche) im Vergleich zu 5 bis 10\% unter statischen Bedingungen werden von einer weiteren Studie berichtet [15]. Der offensichtliche Unterschied zu den Ergebnissen unserer Arbeit und den Ergebnissen von Edwards u. Mitarb. [16] erklärt sich aus der Methode, mit der zwischen vitalen und nicht-vitalen Zellen unterschieden wird. Tote Zellen wurden mit Ethidium-Homodimer-1, welches die intakte Zellmembran lebender Zellen nicht passieren kann, detektiert. Allerdings kann dieser Farbstoff in Zellen gelangen, deren Membran nur vorübergehend geschädigt war. Die Zahl der so markierten Zellen kann die Zahl der nekrotischen Zellen, wie sie in unserer Studie mit PI gemessen wurde, deutlich übersteigen.

Stickstoffmonoxid (NO) ist ein bedeutender Bestandteil des extrazellulären Milieus, der das Ausmaß an Apoptose in ATII-Zel- len beeinflussen kann. Es wird sowohl mit pro- als auch mit antiapoptotischen Effekten in Zusammenhang gebracht [23]. Edwards u. Mitarb. [16] konnten erstmalig nachweisen, dass ein NO-Donator (S-nitroso-N-acetyl-D,L-penicillamine [SNAP]) mechanisch induzierte Apoptose in ATII-Zellen hemmt. Die Autoren sehen eine physiologische Funktion von Alveolarmakrophagen darin, über die Freisetzung von NO die ATII-Zellen vor mechanisch induzierter Apoptose zu schützen. Wir konnten belegen, dass auch das physiologische Substrat der NOS, L-Arginin, mechanisch induzierte Apoptose von ATII-Zellen in vergleichbarer Weise verhindert. Diese Beobachtung lässt vermuten, dass NO in unseren Versuchen durch eine NOS der ATII-Zellen freigesetzt wurde. Die myokardiale Caspase-3 wird durch endogenes NO inhibiert [24], so dass auch in unserem Modell Caspase-Inhibition durch NO einen möglichen Mechanismus darstellt, der den antiapoptotischen Effekt von L-Arginin erklären könnte. Die induzierbare NOS (iNOS), die durch inflammatorische Zytokine oder LPS induziert wird [25], könnte ebenso durch mechanische Dehnung induziert werden. Alveoläre Dehnung durch mechanische Beatmung konnte in verschiedenen experimentellen Modellen mit alveolärer NO-Freisetzung in Zusammenhang gebracht werden $[26,27]$. Auch klinische Daten von beatmeten Patienten mit akuter Lungenschädigung demonstrieren einen Zusammenhang zwischen alveolärer Dehnung und NO-Freisetzung [28]. Insgesamt unterstützen diese Ergebnisse unsere Hypothese, dass starke Dehnung alveolärer Strukturen ATII Zell-Apoptose induziert und gleichzeitig dazu, mit der Freisetzung von NO, einen antiapoptotischen Mediator stimuliert.

Weitere Gruppen von Substanzen, deren antiapoptotische Wirkung auf ATII-Zellen nachgewiesen ist, stellen die ACE-Hemmer und die Angiotensin-III-Rezeptorantagonisten dar. Beide Substanzgruppen beeinflussen Apoptose beispielsweise an Herzmuskelzellen, renalen Tubulsepithelien und vaskulären glatten Muskelzellen [29-31]. Sie inhibieren Fas-Ligand induzierte Apoptose von ATII-Zellen [32]. In unserem Modell inhibierte der ACE-Hemmer Captopril ebenso die mechanisch induzierte Apoptose von ATII-Zellen. Da in den Überständen der gedehnten Zellen Angiotensin-II nicht nachweisbar war, müssen wir vermuten, dass die Umwandlung von Angiotensin-I zu Angiotensin-II an der Auslösung der mechanisch induzierten Apoptose nicht beteiligt ist. Vielmehr könnte der ACE-Hemmer auch als Inhibitor anderer Serinproteasen wirken. Außerdem könnten die antiapoptotischen Effekte von NO und ACE-Hemmern auch im Zusammenhang stehen: Im kardiovaskulären System werden die Wirkungen von ACE-Hemmern zumindest teilweise über die Verhinderung des enzymatischen Abbaus von Bradykinin vermittelt. Bradykinin aktiviert in Endothelzellen die endotheliale NOS und kann dadurch zur NO-Freisetzung führen [33].

Mit beiden von uns vorgenommenen pharmakologischen Interventionen konnte die mechanisch induzierte Apoptose, nicht jedoch die Nekrose beeinflusst werden. Dies legt die Vermutung nahe, dass Captopril und L-Arginin den Ablauf der Apoptose beeinflussen, ohne jedoch effektiv zu sein, wenn die mechanisch induzierte Zellmembranschädigung einen kritischen Punkt überschritten hat, was dann zu nekrotischem Zelltod führt. 


\section{Literatur}

${ }^{1}$ Wirtz HR, Dobbs LG. Calcium mobilization and exocytosis after one mechanical stretch of lung epithelial cells. Science 1990; 250: $1266-1269$

2 Wirtz HR, Dobbs LG. The effects of mechanical forces on lung functions. Respir Physiol 2000; 119: 1-17

${ }^{3}$ Sanchez-Esteban J, Cicchiello LA, Wang Y et al. Mechanical stretch promotes alveolar epithelial type II cell differentiation. J Appl Physiol 2001; 91: 589-595

${ }^{4}$ Edwards YS, Sutherland LM, Power JH et al. Cyclic stretch induces both apoptosis and secretion in rat alveolar type II cells. FEBS Lett 1999; 448: $127-130$

${ }^{5}$ Vlahakis NE, Hubmayr RD. Invited review: plasma membrane stress failure in alveolar epithelial cells. J Appl Physiol 2000; 89: 2490 - 2496; discussion 2497

${ }^{6}$ Tschumperlin DJ, Margulies SS. Equibiaxial deformation-induced injury of alveolar epithelial cells in vitro. Am J Physiol 1998; 275: L1173- 1183

${ }^{7}$ Tschumperlin DJ, Oswari J, Margulies AS. Deformation-induced injury of alveolar epithelial cells. Effect of frequency, duration, and amplitude. Am J Respir Crit Care Med 2000; 162: 357-362

8 Ventilation with lower tidal volumes as compared with traditional tidal volumes for acute lung injury and the acute respiratory distress syndrome. The Acute Respiratory Distress Syndrome Network. N Engl J Med 2000; 342: 1301 - 1308

${ }^{9}$ Amato MB, Barbas CS, Medeiros DM et al. Effect of a protective-ventilation strategy on mortality in the acute respiratory distress syndrome. N Engl J Med 1998; 338: 347 - 354

${ }^{10}$ Uhlig S. Ventilation-induced lung injury and mechanotransduction: stretching it too far? Am J Physiol Lung Cell Mol Physiol 2002; 282: L892-896

${ }^{11}$ Uhlig U, Haitsma JJ, Goldmann T et al. Ventilation-induced activation of the mitogen-activated protein kinase pathway. Eur Respir J 2002; 20: $946-956$

12 Schneider M, Hartung T. Induction of the chemokines IL-8 and MCP-1 in human whole blood by a cell-lysate of human fibroblast cells. Immunol Lett 2001; 75: $163-165$

${ }^{13}$ Bardales RH, Xie SS, Schaefer RF et al. Apoptosis is a major pathway responsible for the resolution of type II pneumocytes in acute lung injury. Am J Pathol 1996; 149: 845-852

${ }^{14}$ Hallman M, Spragg R, Harrell JH et al. Evidence of lung surfactant abnormality in respiratory failure. Study of bronchoalveolar lavage phospholipids, surface activity, phospholipase activity, and plasma myoinositol. J Clin Invest 1982; 70: 673-683

${ }^{15}$ Oswari J, Matthay MA, Margulies SS. Keratinocyte growth factor reduces alveolar epithelial susceptibility to in vitro mechanical deformation. Am J Physiol Lung Cell Mol Physiol 2001; 281: L1068 - 1077

${ }^{16}$ Edwards YS, Sutherland LM, Murray AW. NO protects alveolar type II cells from stretch-induced apoptosis. A novel role for macrophages in the lung. Am J Physiol Lung Cell Mol Physiol 2000; 279: L1236-1242

17 Dobbs LG, Gonzalez R, Williams MC. An improved method for isolating type II cells in high yield and purity. Am Rev Respir Dis 1986; 134: $141-145$
${ }^{18}$ Wirtz HR, Schmidt M. Acute influence of cigarette smoke on secretion of pulmonary surfactant in rat alveolar type II cells in culture. Eur Respir J 1996; 9: 24-32

${ }^{19}$ Willner J, Vordermark D, Schmidt M et al. Secretory activity and cell cycle alteration of alveolar type II cells in the early and late phase after irradiation. Int J Radiat Oncol Biol Phys 2003; 55: 617-625

20 Tschumperlin DJ, Margulies SS. Alveolar epithelial surface area-volume relationship in isolated rat lungs. J Appl Physiol 1999; 86: 2026-2033

${ }^{21}$ Okano H, Shiraki K, Inoue H et al. Cellular FLICE/caspase-8-inhibitory protein as a principal regulator of cell death and survival in human hepatocellular carcinoma. Lab Invest 2003; 83: 1033 - 1043

22 Matute-Bello G, Winn RK, Jonas M et al. Fas (CD95) induces alveolar epithelial cell apoptosis in vivo: implications for acute pulmonary inflammation. Am J Pathol 2001; 158: 153 - 161

${ }^{23}$ Janssen YM, Soultanakis R, Steece K et al. Depletion of nitric oxide causes cell cycle alterations, apoptosis, and oxidative stress in pulmonary cells. Am J Physiol 1998; 275: L1100 - 1109

${ }^{24}$ Mital S, Barbone A, Addonizio LJ et al. Endogenous endothelium-derived nitric oxide inhibits myocardial caspase activity: implications for treatment of end-stage heart failure. J Heart Lung Transplant 2002; 21: $576-585$

${ }^{25}$ Guo FH, De Raeve HR, Rice TW et al. Continuous nitric oxide synthesis by inducible nitric oxide synthase in normal human airway epithelium in vivo. Proc Natl Acad Sci U S A 1995; 92: 7809-7813

${ }^{26}$ Bannenberg GL, Gustafsson LE. Stretch-induced stimulation of lower airway nitric oxide formation in the guinea-pig: inhibition by gadolinium chloride. Pharmacol Toxicol 1997; 81: 13 - 18

${ }^{27}$ Hammerschmidt S, Schiller J, Kuhn $\mathrm{H}$ et al. Influence of tidal volume on pulmonary NO-release, tissue lipid peroxidation and surfactant phospholipids. Biochim Biophys Acta 2002; 1639: 16-27

28 Gessner C, Hammerschmidt S, Kuhn $\mathrm{H}$ et al. Exhaled breath condensate nitrite and its relation to tidal volume in acute lung injury. Chest 2003; 124: 1046 - 1052

${ }^{29}$ Kelly DJ, Cox AJ, Tolcos M et al. Attenuation of tubular apoptosis by blockade of the renin-angiotensin system in diabetic Ren-2 rats. Kidney Int 2002; 61: 31 - 39

${ }^{30}$ Yu G, Liang X, Xie X et al. Diverse effects of chronic treatment with losartan, fosinopril, and amlodipine on apoptosis, angiotensin II in the left ventricle of hypertensive rats. Int J Cardiol 2001; 81: 123-129; discussion $129-130$

${ }^{31}$ Nagashima H, Sakomura Y, Aoka Y et al. Angiotensin II type 2 receptor mediates vascular smooth muscle cell apoptosis in cystic medial degeneration associated with Marfan's syndrome. Circulation 2001; 104: I282-287

32 Uhal BD, Gidea C, Bargout R et al. Captopril inhibits apoptosis in human lung epithelial cells: a potential antifibrotic mechanism. Am J Physiol 1998; 275: L1013-1017

${ }^{33}$ Kitakaze M, Node K, Takashima S et al. Cellular mechanisms of cardioprotection afforded by inhibitors of angiotensin converting enzyme in ischemic hearts: role of bradykinin and nitric oxide. Hypertens Res 2000; $23: 253-259$ 